\title{
Neutralino Dark Matter in Gauge Mediation in Light of CDMS-II
}

\author{
Masahiro Ibe $^{1}$ and Tsutomu T. Yanagida ${ }^{2}$ \\ ${ }^{1}$ Department of Physics and Astronomy, University of California, Irvine, California 92697, USA \\ ${ }^{2}$ Institute for the Physics and Mathematics of the Universe, University of Tokyo, Kashiwa 277-8568, Japan.
}

(Dated: June 28, 2018)

\begin{abstract}
A recent observation of the two candidate events of the dark matter recoiling at CDMS-II is suggestive of dark matter with a mass not far above $100 \mathrm{GeV}$. We propose a model of gauge mediated supersymmetry breaking where the lightest neutralino is identified as dark matter which may provide the observed signals.
\end{abstract}

\section{Introduction}

For more than 70 years, dark matter has eluded direct detection, and its nature still remains unclear. However, a recent observation of the two candidate events of the dark matter recoiling at CDMS-II may be providing an important hint of the nature of dark matter [1]. Especially, the recoil energies detected at $12.3 \mathrm{keV}$ and $15.5 \mathrm{keV}$ suggest that the mass of dark matter is not so heavier than $100 \mathrm{GeV}$.

From the theory side in the era of the LHC, the most interesting candidate of dark matter is the lightest neutralino in the supersymmetric standard model (SSM). So far, there have been a lot of works on the direct detection of the neutralino dark matter scenario [2].

From the point of view of supersymmetric model building, however, the neutralino dark matter with a mass not so far above $100 \mathrm{GeV}$ has some tensions. For example, in gravity mediation, all the superparticles are expected to have comparable masses. Such a rather light spectrum, however, predicts too light higgs particles. Besides, in gravity mediation, it is rather difficult to suppress the supersymmetric contributions to flavor changing neutral current (FCNC) processes. ${ }^{1}$ On the other hand, in most of the models with gauge mediation, the gravitino is lighter than the lightest neutralino, and hence, the lightest neutralino is no more the dark matter candidate, although the FCNC problem is naturally solved.

In this paper, we propose a model with gauge mediation where the gravitino is heavier than the lightest neutralino, while the masses of the sfermions are dominated by the gauge mediation effects.

\section{Hierarchical gauge mediation}

In order for the lightest neutralino with a mass not so far above $100 \mathrm{GeV}$ to be the lightest superparticle,

\footnotetext{
${ }^{1}$ Both the problems in gravity mediation can be ameliorated in large cutoff supergravity models in Ref. 3]. The interpretation of the CDMS-II results in large cutoff supergravity will be disscussed elsewhere [4].
}

the gravitino mass should be heavier than the lightest neutralino, i.e.,

$$
m_{3 / 2}=\frac{F}{\sqrt{3} M_{\mathrm{PL}}} \gtrsim 100 \mathrm{GeV} .
$$

Here, $F$ denotes a supersymmetry breaking vacuum expectation value and $M_{\mathrm{PL}}=2.4 \times 10^{18} \mathrm{GeV}$ is the reduced Planck scale. Thus, the supersymmetry breaking expectation value is larger than,

$$
F \gtrsim 4 \times 10^{20} \mathrm{GeV}^{2}
$$

On the other hand, the sfermion masses should be dominated by the gauge mediated contributions, so that the flavor violating masses from gravity mediation are relatively suppressed, i.e.

$$
m_{\text {scalar }}^{(\text {GMSB })} \gg m_{3 / 2} \gtrsim 100 \mathrm{GeV} \text {. }
$$

In this study, we assume that the gravity mediation contribution is less than about $1 \%$, i.e.,

$$
m_{3 / 2}^{2} / m_{\mathrm{scalar}}^{(\mathrm{GMSB}) 2} \lesssim 0.01
$$

or equivalently,

$$
m_{\mathrm{scalar}}^{(\mathrm{GMSB})} \gtrsim 1 \mathrm{TeV} .
$$

Put it all together, we require a hierarchical spectrum,

$$
\left\{m_{\text {gaugino }}^{\text {(GMSB })}, \mu_{H}\right\} \lesssim m_{3 / 2} \ll m_{\text {scalar }}^{(\text {GMSB })}
$$

where $\mu_{H}$ denotes the supersymmetric higgs mixing parameter $(\mu$-term). Hereafter, we assume that the sfermion masses are not far above $O(1) \mathrm{TeV}$ to avoid the large hierarchy problem.

Can such a hierarchical spectrum be realized in models with gauge mediation? In fact, it is generic that the gaugino masses are suppressed compared with the sfermion masses in R-symmetric gauge mediation models 5 ].

To see the suppression explicitly, let us consider a model of gauge mediation developed in Refs. [6, 7], where the messenger fields $\psi, \bar{\psi}, \psi^{\prime}$ and $\bar{\psi}^{\prime}$ couple to a supersymmetry breaking chiral superfield, $S=F_{S} \theta^{2}$, in the superpotential,

$$
W=S \psi \bar{\psi}+M_{\mathrm{mess}} \psi \bar{\psi}^{\prime}+M_{\mathrm{mess}} \psi^{\prime} \bar{\psi}+M_{\not R} \psi^{\prime} \bar{\psi}^{\prime} .
$$


Here, $M_{\text {mess }}$ and $M_{\not h}$ are mass parameters. The above superpotential possesses the R-symmetry in the limit of vanishing $M_{\not R}$. In the followings, we assume $F_{S}=F$ for simplicity, although we can extend our analysis for more generic cases with $F_{S}<F$ straightforwardly.

In this model, the sfermion masses are given by,

$$
m_{\mathrm{scalar}}^{2} \simeq \sum_{a=1,2,3} 2 C_{a}\left(\frac{\alpha_{a}}{4 \pi}\right)^{2}\left(\frac{F}{M_{\mathrm{mess}}}\right)^{2},
$$

where $\alpha_{a}$ denotes the fine structure constant of the each SSM gauge groups, and $C_{a}$ is an order one coefficient which depends on the group representations of the messenger fields. Here, we have assumed that the mass parameter $M_{\not h}$ is smaller than $M_{\text {mess }}$, which will be our main concern in the following discussion. From the supersymmetry breaking scale in Eq. (2), the sfermion masses in the $\mathrm{TeV}$ range imply

$$
M_{\text {mess }} \simeq 10^{15} \mathrm{GeV} .
$$

On the other hand, the gaugino masses $(a=1,2,3)$ are given by [8],

$$
m_{a} \simeq b_{a} \frac{\alpha_{a}}{4 \pi} \times F \times \frac{\partial}{\partial S} \log \left[\operatorname{det} M_{\psi}\right],
$$

where $b_{a}$ denotes an order one coefficient which also depends on the group representations of the messenger fields. Here, the mass matrix $M_{\psi}$ is defined by,

$$
M_{\psi}=\left(\begin{array}{cc}
S & M_{\text {mess }} \\
M_{\text {mess }} & M_{\not h}
\end{array}\right),
$$

and its determinant is given by,

$$
\operatorname{det} M_{\psi}=S M_{\not h}-M_{\text {mess }}^{2} .
$$

From Eqs. (10) and (12), we immediately find that the gaugino mass is vanishing in the limit of $M_{\not R} \rightarrow 0$, even if the supersymmetry breaking chiral field $S$ obtains a spontaneous R-symmetry breaking scalar expectation value from a supersymmetry breaking sector, i.e. $S=M_{R}+F_{S} \theta^{2}{ }^{2}$ With non-vanishing $M_{\not h}$, the gaugino masses are given by,

$$
m_{a} \simeq b_{a} \frac{\alpha_{a}}{4 \pi} \times \frac{F}{M_{\mathrm{mess}}} \times \frac{M_{\not R}}{M_{\mathrm{mess}}} .
$$

2 Strictly speaking, the gauginos obtain masses which are suppressed by $\left|F / M_{\text {mess }}^{2}\right|^{2}$ than the sfermion masses in Eq. (8) even in the limit of $M_{\not R} \rightarrow 0$. However, they are negligibly small for $M_{\text {mess }} \simeq 10^{15} \mathrm{GeV}$. Those gaugino masses can be important when the messenger scale is as low as $M_{\text {mess }}=O(100) \mathrm{TeV}$ for $F_{S} \ll F$. Even in this case, the gaugino masses are still smaller than the sfermions [9], and hence, we may explain the hierarchical spectrum without introducing $M_{\not R}$. A detailed analysis of this case will be given elsewhere.
Therefore, by choosing an appropriate R-breaking mass $M_{\not h}$, we can realize suppressed gaugino masses compared with the sfermion masses.

The suppressed gravitino mass compared with the sfermion masses is also beneficial to explain the suppressed $\mu$-term. That is, the $\mu$-term can be generated by the Giudice-Masiero mechanism [10], where the $\mu$-term originates from a term in the Kalher potential, ${ }^{3}$

$$
K=\frac{c_{H} S^{\dagger}}{\sqrt{3} M_{\mathrm{PL}}} H_{u} H_{d}+\text { h.c. . }
$$

Here, $c_{H}$ is an order one coefficient, and $H_{u, d}$ denote the Higgs doublet superfields. Then, the resultant $\mu$-term is given by,

$$
\mu_{H}=c_{H} \times m_{3 / 2} .
$$

Thus, the suppressed gravitino mass provides the suppressed $\mu$-term.

\section{Discussions}

In the previous section, we have proposed a model with the lightest neutralino dark matter which are free from the FCNC problem; a gauge mediation model with a hierarchical spectrum in Eq. (5). The gravitino mass is in between the lightest neutralino mass and the sfermion masses. In this model, for example, we may obtain a following parameter set,

$$
\begin{aligned}
& m_{1}=75 \mathrm{GeV}, m_{2}=350 \mathrm{GeV}, m_{3}=800 \mathrm{GeV}, \\
& \mu_{H}=125 \mathrm{GeV}, \tan \beta=10, m_{\text {scalar }}=2 \mathrm{TeV},
\end{aligned}
$$

where the parameters are given at the renormalization scale around the electroweak scale. For simplicity, we have assigned the same masses to all the squarks and sfermions, although they are not relevant for the direct detection rate below. With this mass parameter set, the lightest neutralino has a mass $m_{\chi}=59 \mathrm{GeV}$ and gets sizable higgsino components. The expected number of events at direct detection by Ge detectors is $1.16 \times 10^{-2} /$ day $/ \mathrm{kg},\left(\sigma_{\chi-p}^{S I}=2.9 \times 10^{-44} \mathrm{~cm}^{2}\right)$, which is consistent with the detection of the two candidate events in CDMS-II [1], while the relic density of dark matter is consistent with the WMAP observation [12]. ${ }^{4}$

3 We may also obtain a similar size of $\mu$-term via a Kahler potential [1],

$$
K=c_{H}^{\prime} H_{u} H_{d}+\text { h.c. , }
$$

which gives $\mu_{H}=c_{H}^{\prime} m_{3 / 2}$.

${ }^{4}$ In this analysis, we have used micrOMEGAs2.1 [13]. The annihilation process is dominated by an $s$-channel pole exchange of the light higgs boson with a mass of $117 \mathrm{GeV}$. For a heavier neutralino with $m_{\chi}>m_{Z, W}$, the annihilation process is dominated by the modes into $W^{+} W^{-}$and $Z Z$ bosons which are allowed by the higgsino components of the lightest neutralino. 
Several comments are in order. In the above parameter set, we have not assumed the so-called GUT (grand unified theory) relations between the mass parameters. ${ }^{5}$ In this model, such a spectrum without the GUT relation can be easily realized in a GUT consist manner. For example, we may introduce coupling constants between the messengers and the supersymmetry breaking field which depend on the vacuum expectation value for spontaneous breaking of the GUT gauge group. In this case, the coupling constants between messenger fields and the supersymmetry breaking field do not necessarily satisfy the GUT relation. Thus, for example, the mass ratio $m_{1} / m_{2} \simeq 0.21$ in Eq. (17) can be realized when the ratios of the coupling constants of $S Q_{M} \bar{Q}_{M}, S \bar{U}_{M} U_{M}$ and $S \bar{E}_{M} E_{M}$ interaction in Eq. (7) are around $1: 0.4: 0.4$, while keeping the common messenger masses. Here, $\left(Q_{M}, \bar{U}_{M}, \bar{E}_{M}\right)$ and its conjugate representations denote messenger fields of $\mathbf{1 0}+\mathbf{1 0} *$ representations in terms of the $S U(5)$ GUT gauge group. ${ }^{6}$

As another example, we may also introduce messenger fields which do not consist of the complete representations of the GUT gauge groups, such as the messenger fields belonging to the adjoint representations of $S U(3)$ and $S U(2)$ gauge groups in the SSM which would be in the adjoint representation of the $S U(5)$ GUT gauge group. ${ }^{7}$ From this messenger sector, there is no contributions to the $m_{1}$ while $m_{2,3}$ obtain non-vanishing gaugino masses. Thus, the gaugino masses without the GUT relation can be explained by introducing multiple messenger fields such as $\mathbf{5}+\mathbf{5}^{*}$ representations in terms of the $S U(5)$ GUT gauge group with the above adjoint representations [15] (see also Ref. 16] for a recent related discussion on the adjoint messengers). ${ }^{8}$

Electroweak symmetry breaking may require careful tuning between mass parameters. In this model, the supersymmetry breaking masses of the Higgs doublets are in the TeV range at the mediation scale. For a successful electroweak symmetry breaking, the one of them should be in a similar size of the $\mu$-term at the electroweak scale. That is, the supersymmetry breaking mass at

${ }^{5}$ The gaugino mass not satisfying the GUT relation is not absolutely necessary to explain the CDMS-II result.

${ }^{6}$ As an interesting prediction, the mass ratio $m_{1} / m_{2}$ is correlated to the mass ratio between right-handed and left-handed sleptons.

7 The introduction of the adjoint representations of the $S U(3)$ and $S U(2)$ gauge groups does not spoil the coupling unification, though the unification scale is affected [14]. As long as the messenger scale is close enough to the so-called GUT scale, $M_{\mathrm{GUT}} \simeq 10^{16} \mathrm{GeV}$, however, the change of the unification scale is small.

${ }^{8}$ When the mediation scale in Eq. (9) is close to the GUT scale, the gaugino masses also get contributions from the remaining heavier components in the adjoint representations of the $S U(5)$ GUT gauge group. In this case, we may not need to introduce multiple messenger fields to explain the relatively small $m_{1}$ than $m_{2,3}$. the mediation scale should be cancelled by the renormalization group contributions which mainly come from the stop mass contributions. Furthermore, the GiudiceMasiero mechanism in Eq. (15) generates the supersymmetry breaking Higgs mixing parameter $(B \mu$-term),

$$
B \mu=c_{H} m_{3 / 2}^{2} .
$$

Thus, the $B \mu$-term is also suppressed than the Higgs soft masses, which tends to predict rather large $\tan \beta$. A further consistency check of electroweak symmetry breaking will be discussed elsewhere.

The cosmic abundance of the gravitino with a mass in the hundreds $\mathrm{GeV}$ range is strictly restricted by the constraints on the effects on the Big-Bang-Nucleosynthesis $(\mathrm{BBN})$. As discussed in Ref. [17], the reheating temperature after the primordial inflation is constrained to be lower than $10^{6-7} \mathrm{GeV}$ for $m_{3 / 2}=O(100) \mathrm{GeV}$ to suppress the gravitino abundance. The reheating temperature in this range is too low to explain the baryon asymmetry via Leptogenesis [18]. ${ }^{9}$ Therefore, it is a non-trivial question whether there's a consistent scenario of cosmology with this model including the baryon asymmetry.

The $\mathrm{CP}$ violations of the gaugino masses and the $\mu$ and $B \mu$-terms can be unacceptably large when we introduce multiple messengers. In this case, the phases of the gaugino masses are no more universal, which cannot be rotated away by using the definitions of the fields. ${ }^{10} \mathrm{Be}-$ sides, the phases appearing in $\mu$-term and $B \mu$-term cannot be rotated away. Those CP-violating phases lead to, for example, too large electric dipole moment. A possible way out to this problem is to consider spontaneous $\mathrm{CP}$ violation 21], which eliminate all the CP-phases by symmetry while providing the CKM phase after spontaneous $\mathrm{CP}$ breaking.

Finally, we comment on other possibilities to realize the neutralino dark matter in gauge mediation. As discussed in Refs. 22, 23, 24], the neutralino dark matter is also possible in gauge mediation when the gravity mediated supersymmetry breaking effects are suppressed by the so called sequestering mechanisms [25, 26].$^{11}$ In those attempts, we do not need a hierarchy between the gaugino masses and the sfermion masses to suppress the flavor violating scalar masses (see details in the appendix).

\footnotetext{
9 The non-thermal Leptogenesis works for a relatively low reheating temperature as low as $10^{6-7} \mathrm{GeV}[19,[20]$.

10 As briefly mentioned above, we may have a desired hierarchical spectrum with $M_{\not h}=0$ for $M_{\text {mess }}=O(100) \mathrm{GeV}$. In such an $\mathrm{R}$-symmetric gauge mediation model, we can rotate away the phases of the gauginos.

${ }^{11}$ See also Refs. 27, 28, 29] for the later development of the con-
} formal sequestering mechanisms. 


\section{Acknowledgements}

The work T.T.Y. was supported in part by World Premier International Research Center Initiative (WPI Initiative), MEXT, Japan.

\section{Appendix: Gauge mediation with sequestered gravity mediation}

In this appendix, we give a bare bones outline of a gauge mediated model with sequestered gravity mediation which provides the rather light lightest neutralino.

To be specific, we consider the vector-like supersymmetry breaking model based on an $S U(2)$ gauge theory with four fundamental representation fields $Q_{i}(i=1, \cdots, 4)$ and six singlet fields $Z_{i j}=-Z_{j i}(i, j=1, \cdots, 4)$ [30, 31]. In this model, the SUSY is dynamically broken when the $Q$ 's and $Z$ 's couple in the superpotential,

$$
W=\lambda_{i j} Z_{i j} Q_{i} Q_{j},(i<j) \text {, }
$$

where $\lambda_{i j}$ denotes coupling constants. The supersymmetry is broken as a result of the tension between the $F$-term conditions of $Z$ 's and $Q$ 's.

According to Ref. [27], we can extend the above supersymmetry breaking model to a model with conformal sequestering by adding appropriate number of gauge charged fields with an appropriate gauge group extension. The extended model flows to the original supersymmetry breaking model below the mass scale of the newly added charged fields, $M_{\text {seq }}$, which is explicit breaking to the conformal symmetry at the higher energy scale. Then, the gravity mediation effects are suppressed by,

$$
m_{\text {scalar }}^{\text {(gravity)2 }} \sim\left(\frac{M_{\text {seq }}}{M_{\mathrm{PL}}}\right)^{\beta^{\prime}} \times m_{3 / 2}^{2},
$$

when the model is in the vicinity of the infrared fixed point of the extended model around the Planck scale. Here, $\beta^{\prime}$ denotes the derivative of the beta function of the gauge coupling constant with respect to the fine-structure constant and it is expected to be of the order of one when the model is strongly interacting. Thus, for example, $M_{\text {seq }} \simeq 10^{11} \mathrm{GeV}$ provides enough suppressions to the gravity mediation effects for $m_{3 / 2}=O(100) \mathrm{GeV}$.

Now, let us consider to mediate supersymmetry breaking to the SSM sector. For that purpose, we again introduce the messenger sector used in the text, but here, we assume that $M_{\not k}=0$. Besides, we treat the chiral field $S$ not as a spurious field as in the text, but as a dynamical field which possesses a cubic term in the superpotential. Altogether, the messenger sector is given by,

$$
W=S \psi \bar{\psi}+M_{\mathrm{mess}} \psi \bar{\psi}^{\prime}+M_{\mathrm{mess}} \psi^{\prime} \bar{\psi}+\frac{f}{3} S^{3}
$$

where $f$ denotes a coupling constant. Furthermore, to connect the messenger sector to the supersymmetry breaking sector, we use a mechanism in Ref. [32] where we gauge a $U(1)$ subgroup of the global symmetry in the supersymmetry breaking sector ${ }^{12}$ and introduce a pair of $U(1)$ gauge charged superfields $E$ and $\bar{E} .{ }^{13}$ The supersymmetry breaking effects are transmitted to $S$ via a coupling,

$$
W=k S E \bar{E}
$$

where $k$ is a coupling constant. As shown in Ref. [32], the $S$ field obtains a negative supersymmetry breaking mass squared, $m_{S}^{2}$, at the two-loop level which destabilize the origin of $S$. As a result, the R-symmetry and the supersymmetry in the messenger sector are broken by,

$$
\langle S\rangle=\frac{m_{S}}{f}, \quad\left\langle F_{S}\right\rangle=\frac{m_{S}^{2}}{f} .
$$

The gaugino masses and the sfermion masses are, then, given by,

$$
\begin{aligned}
m_{a} & \simeq b_{a} \frac{\alpha_{a}}{4 \pi} \frac{\langle S\rangle}{M_{\text {mess }}}\left|\frac{\left\langle F_{S}\right\rangle}{M_{\text {mess }}^{2}}\right|^{2} \frac{\left\langle F_{S}\right\rangle}{M_{\text {mess }}}, \\
m_{\text {scalar }}^{2} & \simeq \sum_{a} 2 C_{a}\left(\frac{\alpha_{a}}{4 \pi}\right)^{2}\left(\frac{\left\langle F_{S}\right\rangle}{M_{\text {mess }}}\right)^{2} .
\end{aligned}
$$

Here, we have assumed $\langle S\rangle \lesssim M_{\text {mess }}$. Thus, when $\langle S\rangle$, $M_{\text {mess }}, \sqrt{F_{S}}$ are all in the hundreds $\mathrm{TeV}$ range, we can realize

$$
m_{a}, m_{\mathrm{scalar}}=O(100) \mathrm{GeV}-O(1) \mathrm{TeV},
$$

although the gaugino masses tend to be suppressed compared to the scalar masses. ${ }^{14}$

It should be noted that the scalar masses (especially the slepton masses) can be smaller than ones in the model discussed in the text, since the flavor-violating gravity mediated effects are sequestered away. Therefore, this

12 We impose a global $S U(4)$ symmetry of the supersymmetry breaking sector in the limit of the $U(1)$ gauge coupling vanishing. Then, as pointed out in Ref. [27], we have sequestering. This sequestering will be maintained for a non-vanishing $U(1)$ gauge coupling as long as the coupling is very small.

13 One may consider to identify $S$ with one of $Z_{i j}$ which is responsible for supersymmetry breaking. However, in the model in Eq. (21), the couplings between $S$ (i.e. $Z$ 's) and the messenger fields are also sequestered and results in too small gaugino mass (see Eq. (24)).

14 The messenger sector without $\psi^{\prime}$ and $\bar{\psi}^{\prime}$ fields with a superpotential

$$
W=S \psi \bar{\psi}+\frac{f}{3} S^{3},
$$

works as well. In this case, the predicted spectrum is no more hierarchical. 
model will be preferred if we discover relatively light sfermions at the collider experiments such as LHC.

Finally, we mention an advantage of this model. This model is impervious to the CP-problem, while the model in the text may suffer from the problem when we introduce multiple messengers. That is, in this class of $\mathrm{R}$-symmetric gauge mediation models, the CP-phases of the coupling constant between the supersymmetry breaking field and the messengers are rotated away even in the case of the multiple messengers.

[1] Z. Ahmed et.al. [The CDMS Collaboration], arXiv:0912.3592 [astro-ph.CO].

[2] For a review, see G. Jungman, M. Kamionkowski and K. Griest, Phys. Rept. 267, 195 (1996) arXiv:hep-ph/9506380.

[3] M. Ibe, K. I. Izawa and T. Yanagida, Phys. Rev. D 71, 035005 (2005) arXiv:hep-ph/0409203.

[4] M. Ibe, K.-I. Izawa,T.T. Yanagida, in preparation.

[5] Z. Komargodski and D. Shih, JHEP 0904, 093 (2009) arXiv:0902.0030 [hep-th]].

[6] K. I. Izawa, Y. Nomura, K. Tobe and T. Yanagida, Phys. Rev. D 56, 2886 (1997) arXiv:hep-ph/9705228;

[7] Y. Nomura and K. Tobe, Phys. Rev. D 58, 055002 (1998) arXiv:hep-ph/9708377.

[8] G. F. Giudice and R. Rattazzi, Nucl. Phys. B 511, 25 (1998) arXiv:hep-ph/9706540.

[9] M. Ibe, K. Tobe and T. Yanagida, Phys. Lett. B 615, 120 (2005) arXiv:hep-ph/0503098.

[10] G. F. Giudice and A. Masiero, Phys. Lett. B 206, 480 (1988).

[11] K. Inoue, M. Kawasaki, M. Yamaguchi and T. Yanagida, Phys. Rev. D 45, 328 (1992).

[12] E. Komatsu et al. [WMAP Collaboration], Astrophys. J. Suppl. 180, 330 (2009) arXiv:0803.0547 [astro-ph]].

[13] G. Belanger, F. Boudjema, A. Pukhov and A. Semenov, Comput. Phys. Commun. 180, 747 (2009) arXiv:0803.2360 [hep-ph]].
[14] C. Bachas, C. Fabre and T. Yanagida, Phys. Lett. B 370, 49 (1996) arXiv:hep-th/9510094.

[15] T. Han, T. Yanagida and R. J. Zhang, Phys. Rev. D 58, 095011 (1998) arXiv:hep-ph/9804228.

[16] E. Dudas, S. Lavignac and J. Parmentier, Nucl. Phys. B 808 (2009) 237 arXiv:0808.0562 [hep-ph]].

[17] M. Kawasaki, K. Kohri, T. Moroi and A. Yotsuyanagi, Phys. Rev. D 78, 065011 (2008) arXiv:0804.3745 [hep$\mathrm{ph}]$.

[18] M. Fukugita and T. Yanagida, Phys. Lett. B 174, 45 (1986).

[19] K. Kumekawa, T. Moroi and T. Yanagida, Prog. Theor. Phys. 92, 437 (1994) arXiv:hep-ph/9405337.

[20] G. Lazarides, R. K. Schaefer and Q. Shafi, Phys. Rev. D 56, 1324 (1997) arXiv:hep-ph/9608256.

[21] G. Hiller and M. Schmaltz, Phys. Rev. D 65, 096009 (2002) arXiv:hep-ph/0201251.

[22] Y. Nomura and K. Suzuki, Phys. Rev. D 68, 075005 (2003) arXiv:hep-ph/0110040.

[23] S. Shirai, F. Takahashi, T. T. Yanagida and K. Yonekura, Phys. Rev. D 78 (2008) 075003 arXiv:0808.0848 [hep$\mathrm{ph}]$.

[24] N. J. Craig and D. R. Green, Phys. Rev. D 79, 065030 (2009) arXiv:0808.1097 [hep-ph]].

[25] L. Randall and R. Sundrum, Nucl. Phys. B 557, 79 (1999) arXiv:hep-th/9810155.

[26] M. Luty and R. Sundrum, Phys. Rev. D 67, 045007 (2003) arXiv:hep-th/0111231.

[27] M. Ibe, K. I. Izawa, Y. Nakayama, Y. Shinbara and T. Yanagida, Phys. Rev. D 73, 015004 (2006) arXiv:hep-ph/0506023;

[28] M. Ibe, K. I. Izawa, Y. Nakayama, Y. Shinbara and T. Yanagida, Phys. Rev. D 73, 035012 (2006) arXiv:hep-ph/0509229.

[29] M. Schmaltz and R. Sundrum, JHEP 0611, 011 (2006) arXiv:hep-th/0608051.

[30] K. I. Izawa and T. Yanagida, Prog. Theor. Phys. 95, 829 (1996) arXiv:hep-th/9602180;

[31] K. A. Intriligator and S. D. Thomas, Nucl. Phys. B 473, 121 (1996) arXiv:hep-th/9603158.

[32] Y. Nomura, K. Tobe and T. Yanagida, Phys. Lett. B 425, 107 (1998) arXiv:hep-ph/9711220. 